Corresponding Author: Luis Tinerfe Hernández Rodríguez

luis.hernandezr@epn.edu.ec

Received: 15 November 2017

Accepted: 5 January 2018

Published: 4 February 2018

Publishing services provided

by Knowledge E

(c) Jorge Ricardo Vintimilla Jaramillo and Luis Tinerfe Hernández Rodríguez. This article is distributed under the terms of the Creative

Commons Attribution License,

which permits unrestricted use and redistribution provided that the original author and source are credited.

Selection and Peer-review under the responsibility of the ESTEC Conference Committee.

\section{S OPEN ACCESS}

\section{Conference Paper}

\section{Columnas Compuestas de Hormigón y Acero SRC, Sujetas a Flexocompresión Biaxial}

\section{Jorge Ricardo Vintimilla Jaramillo and Luis Tinerfe Hernández Rodríguez}

Escuela Politécnica Nacional, Quito, Ecuador

\section{Abstract}

The work presented is based on experimental and theoretical analysis of SRC composite columns subjected to biaxial bending and axial compression, where the specification of American and European code criteria are used to calculate de load bending strength. The computer program to calculate the interaction diagram of biaxial bending and axial compression with inclined neutral axis is made in the software Matlab by using the fiber method, besides, the strength of the specimen is calculated. Users can design new frame sections and check the exist sections. To obtain the displacements and load curve, to calculate load contours and determination of the interaction family curves of the modeled sections. The destructive performance of the round and rectangle composite columns are made in the structures laboratory of EPN to obtain the results such as the buckling displacement at strong, weak and longitudinal direction measured with LVDT'S. Subsequently, the theoretical and experimental analysis results are made to demonstrate the reliability of the numeric model.

\section{Keywords: Composite Columns, Concrete, Steel}

\section{Resumen}

El trabajo consiste en el análisis teórico experimental del comportamiento de columnas mixtas de hormigón reforzadas con perfil de acero (SRC) sujetas a flexocompresión biaxial, se utiliza como base las especificaciones de los códigos americanos y europeos pertinentes para calcular la capacidad de carga y momento nominal. Se elaboró un programa en Matlab por el método de la fibra, para el cálculo del diagrama de interacción a flexocompresión con eje esviado, con el cual se puede realizar los diseños de nuevas secciones, y se puede obtener la curva carga-desplazamiento teórico, calcular los contornos de carga y la determinación de una familia de curvas de interacción de la superficie de falla de la sección modelada. En el laboratorio de estructuras de la EPN se realizaron ensayos destructivos de cuatro columnas mixtas, dos rectangulares y dos circulares para obtener resultados de la capacidad nominal, el desplazamiento por pandeo flexural en la dirección principal y secundaria, medidos 
con LVDT'S. Se obtuvieron los resultados de los esfuerzos que se producen en los miembros. Se hace un análisis y comparación de resultados teóricos y experimentales para comprobar la confiabilidad de los modelos numéricos.

Palabras claves: Columnas compuestas, Hormigón, Acero

\section{Introducción}

El diseño de miembros compuestos tiene que cumplir con factores de seguridad y confianza altos, se requiere una importante ductilidad del miembro, y una relevante capacidad de absorber energía antes de llegar a la rotura. Los códigos de diseño han sido desarrollados en las tres últimas décadas y se modifican periódicamente, por lo que en el presente trabajo se procede a evaluarlos. Se realiza un estudio experimental del caso de columnas rectangulares y circulares mixtas de hormigón armado con perfiles tipo I embebidos (SRC) con la finalidad de comparar las soluciones planteadas en las normativas, se utiliza también un modelo matemático adicional y se obtienen conclusiones al respecto de su resistencia y comportamiento.

Los análisis experimentales realizados a especímenes generan resultados de la resistencia a flexocompresión que son comparados con propuestas teóricas especificadas en los códigos AISC 360 (ANSI/AISC, 2016), EUROCÓDIGO 4 (European Committee for Standardization, 2009), ACl 318 ( $\mathrm{ACl}, 2014)$ con el fin de realizar una validación numérica frente a los resultados experimentales. El método de la AISC se complementa con las observaciones y razonamientos de los autores Segui (Segui, 2013) y McCormac (McCormac, 2013). Se plantea un modelo matemático teórico con el método de la fibra, para convalidar los resultados de la experimentación.

Las columnas a ser estudiadas son mixtas SRC (Steel Reinforced Concrete) y consisten en una sección de hormigón armado reforzado con un perfil "I" de acero estructural embebido. Surge la necesidad de generar un modelo de análisis y diseño mediante un modelo computacional ágil para resolver problemas asociados a la flexocompresión biaxial de las columnas rectangulares y circulares que tengan perfiles de acero embebidos adicionales a barras de refuerzo uniformemente distribuidas en su contorno. Es necesario realizar un modelo experimental para evaluar además si las columnas son suficientemente dúctiles y tenaces. Las columnas son cargadas axialmente en forma excéntrica y el efecto que se presenta tiende a ser de columnas intermedias, con 
condiciones de apoyo empotrado en la base y apoyado en la cabeza, que tiene un factor de longitud efectiva $K=0,70$.

\section{Análisis de Resultados Teóricos y Experimentales}

\subsection{Elaboración de las Probetas de Columnas SRC de Ensayo}

Se construye probetas SRC, dos de sección rectangular y dos de sección circulares con la finalidad de realizar un ensayo de destrucción mediante la aplicación de una carga y momentos biaxiales, para lo cual se realiza una predicción de carga de agotamiento mediante el diagrama de interacción a un determinado ángulo $\beta$ de inclinación del eje neutro. El ensayo se lo ejecuta con un ciclo de carga y descarga, para obtener la rigidez mínima delos miembros
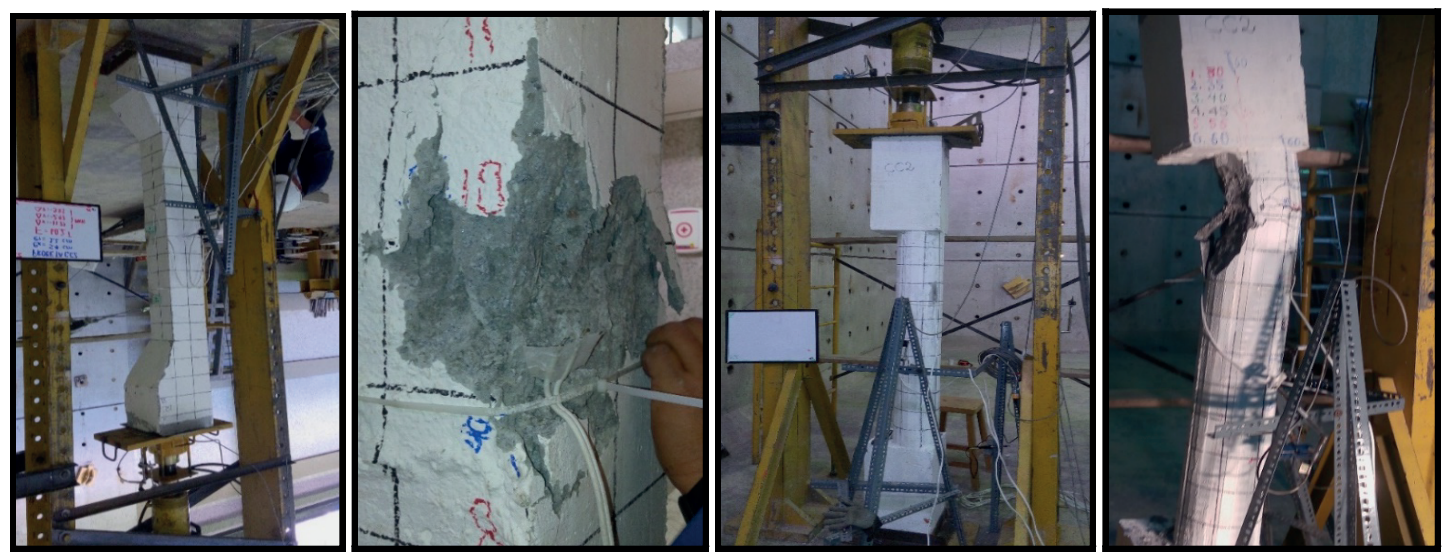

Figura 1: Fotografía de ensayo destructivo de columnas rectangulares y circulares

\subsection{Cálculo del Diagrama de Interacción Teórico}

Para el cálculo del diagrama de interacción por el método de la fibra se trabaja con los datos de la resistencia del hormigón f'c obtenidos a través de ensayos a compresión axial normativos de cilindros de $30 \mathrm{~cm}$ de altura y $15 \mathrm{~cm}$ de diámetro, obtenidas a los 47 días de su fundición. Los valores de fluencia experimental del acero Fy ${ }_{v}$ para varillas y $\mathrm{Fy}_{p}$ para el perfil embebido, se exponen en la Tabla 1, corresponde a ASTM A50 y una deformación unitaria de fluencia de 0.00167. En la tabla 1 CR1 y CR2 es la nomenclatura de las columnas rectangulares, así como $\mathrm{CC}_{1}$ y $\mathrm{CC}_{2}$ corresponden a las columnas circulares.

En la tabla 2 misma ex corresponde a la excentricidad de la carga en el eje $x$, ey corresponde a la excentricidad en el eje $y_{,} P_{e x p}$ es la carga axial nominal obtenida en 
TABLA 1: Resumen de propiedades del hormigón y del acero

\begin{tabular}{|l|c|c|c|c|c|c|}
\hline Probeta & $\mathbf{f}^{\prime} \mathbf{c}$ & Ec & $\mathbf{e}_{\mathbf{c}}$ & Fy varillas $_{\mathbf{v}}$ Fy & Perfil I \\
\hline MPa & MPa & $\mathbf{c m} / \mathbf{c m}$ & MPa & MPa \\
\hline CR1 y CR2 & 32 & 22500 & 0.0022 & 450 & 345 \\
\hline CC1 y CC2 & 38 & 22500 & 0.0022 & 450 & 345 \\
\hline
\end{tabular}

el experimento. Los valores $\mathrm{Mx}_{\text {exp }}$ y $\mathrm{My}_{\text {exp }}$ son lo momentos obtenidos en el ensayo a flexocompresión.

\begin{tabular}{|c|c|c|c|c|c|}
\hline \multirow[t]{2}{*}{ Probeta } & ex & ey & $P_{\text {exp }}$ & $M x_{\exp }$ & $M y_{\text {exp }}$ \\
\hline & m & m & kN & $k N-m$ & $k N-m$ \\
\hline $\mathrm{CR}_{1}$ & 0.053 & 0.071 & 366 & 26.0 & 19.4 \\
\hline $\mathrm{CR}_{2}$ & 0.057 & 0.050 & 560 & 28.0 & 31.9 \\
\hline $\mathrm{CC}_{1}$ & 0.085 & 0.050 & 498 & 24.9 & 42.3 \\
\hline $\mathrm{CC}_{2}$ & 0.054 & 0.012 & 650 & 7.8 & 35.1 \\
\hline
\end{tabular}

Para predecir la carga de falla, ante una combinación de carga axial de compresión Pn y Momentos Biaxiales se trabaja con el diagrama de interacción a un determinado ángulo de esviaje el mismo que fue fijado en la cabeza de las columnas previas a realizar el ensayo.

\subsection{Predicción de Carga Axial y Momentos Flectores}

Se realiza la predicción de carga axial de agotamiento mediante la lectura de datos en los diagramas de interacción calculados utilizando las especificaciones del $\mathrm{ACl} 318$, AISC-LRFD 360-16 y adicionalmente por el método de las FIBRAS, a un eje inclinado $\beta$. Para lograrlo se traza una línea recta inclinada con pendiente e (excentricidad) y que inicie desde el origen de coordenadas hasta intersecar el gráfico del diagrama de interacción respectivo. Se halla la capacidad nominal Pn para cada criterio

La carga nominal obtenida por el método del $\mathrm{ACl} 318$ es $\mathrm{Pn}(\mathrm{I})$, la carga nominal obtenida por el método AISC-LRFD 360 es Pn (II) y la carga nominal obtenida por el método de las fibras es $\mathrm{Pn}$ (III) y se resumen en la tabla 3a, siendo mayores los valores de carga axial obtenidos por el método AISC-LRFD, e intermedios los valores por el método de la fibra. 
TABLA 3: (a) Predicción normativa de carga axial de agotamiento a compresión (kN)

\begin{tabular}{l|c|c|c|c|c|c|c|} 
Criterio & EXP & \multicolumn{2}{|c|}{$\mathrm{ACl}_{318}$} & \multicolumn{2}{c|}{ AISC-LRFD } & \multicolumn{2}{c}{ METODO DE FIBRAS } \\
\hline Probeta & $\mathrm{P}_{\text {exp }} \mathrm{kN}$ & $\mathrm{Pn}(\mathrm{I}) \mathrm{kN}$ & $\mathrm{Pn}(\mathrm{I}) / \mathrm{P}_{\text {exp }}$ & $\mathrm{Pn}(\mathrm{II}) \mathrm{kN}$ & $\mathrm{Pn}(\mathrm{II}) / \mathrm{P}_{\text {exp }}$ & $\mathrm{P} n(\mathrm{III}) \mathrm{kN}$ & $\mathrm{Pn}(\mathrm{III}) / \mathrm{P}_{\text {exp }}$ \\
$\mathrm{CR} 1$ & 366 & 455.8 & 1.25 & 548.4 & 1.50 & 469.7 & 1.28 \\
\hline $\mathrm{CR} 2$ & 560 & 539.3 & 0.96 & 600.8 & 1.07 & 505.0 & 0.90 \\
\hline $\mathrm{CC} 1$ & 498 & 285.8 & 0.57 & 316.8 & 0.64 & 300.0 & 0.60 \\
\hline $\mathrm{CC} 2$ & 650 & 521.3 & 0.80 & 601.5 & 0.93 & 590.0 & 0.91
\end{tabular}

TABLA 3: (b) Predicción normativa de momento nominal (kNm)

\begin{tabular}{|c|c|c|c|c|c|c|c|c|}
\hline \multirow{2}{*}{$\begin{array}{l}\text { Criterio } \\
\text { Probeta }\end{array}$} & \multicolumn{2}{|c|}{ EXPERIMENTAL } & \multicolumn{2}{|c|}{$\mathrm{ACl} 318$} & \multicolumn{2}{|c|}{ AISC-LRFD } & \multicolumn{2}{|c|}{ FIBRAS } \\
\hline & $M \mathrm{x}_{\text {exp }}$ & $M y_{\exp }$ & $\operatorname{Mnx}(1)$ & $\operatorname{Mny}(I)$ & $\operatorname{Mnx}(I I)$ & Mny(II) & $\operatorname{Mnx}(\mathrm{III})$ & Mny(III) \\
\hline CR1 & 26.0 & 19.4 & 48.2 & 43.1 & 52.7 & 47.2 & 35.7 & 41.5 \\
\hline CR2 & 28.0 & 31.9 & 48.9 & 43.8 & 53.1 & 47.1 & 35.8 & 41.9 \\
\hline $\mathrm{CC}_{1}$ & 24.9 & 42.3 & 29.3 & 24.2 & 33.6 & 27.7 & 25.6 & 32.7 \\
\hline $\mathrm{CC}_{2}$ & 7.8 & 35.1 & 28.5 & 23.9 & 35.3 & 28.5 & 25.5 & 32.1 \\
\hline
\end{tabular}

Los momentos experimentales y teóricos (normativos) se muestran en la Tabla 3b, corresponden al estado de flexocompresión biaxial, los valores son calculados en base a la condición de flexión límite en cada dirección. Los momentos nominales obtenidos por el método del $\mathrm{ACl} 318$ son $\mathrm{Mnx}(\mathrm{I})$ y Mny (I), el momento nominal obtenido por el método AISC-LRFD 360-16 es Mnx(II) y Mny (II) para cada dirección y por último el momento nominal obtenido por el método de las fibras son Mnx (III) y Mny (III), siendo mayores los valores obtenidos por el método AISC-LRFD y menores los valores calculados por el método de la fibra.

\subsection{DESARROLLO DEL PROGRAMA POR EL MÉTODO DE LA FIBRA PARA COLUMNAS RECTANGULARES Y CIRCULARES}

El método de la fibra es utilizado para calcular el diagrama de interacción a flexocompresión biaxial en columnas SRC de columnas rectangulares y circulares. Aquí se subdivide a los elementos de la sección transversal de las columnas rectangular en elementos o fibras rectangulares.

La figura 2 representa el esquema de la distribución de las fibras correspondientes de cada material en las zonas de compresión y tracción, se debe tomar en consideración que el hormigón trabaja únicamente en compresión y los aceros en tracción y 


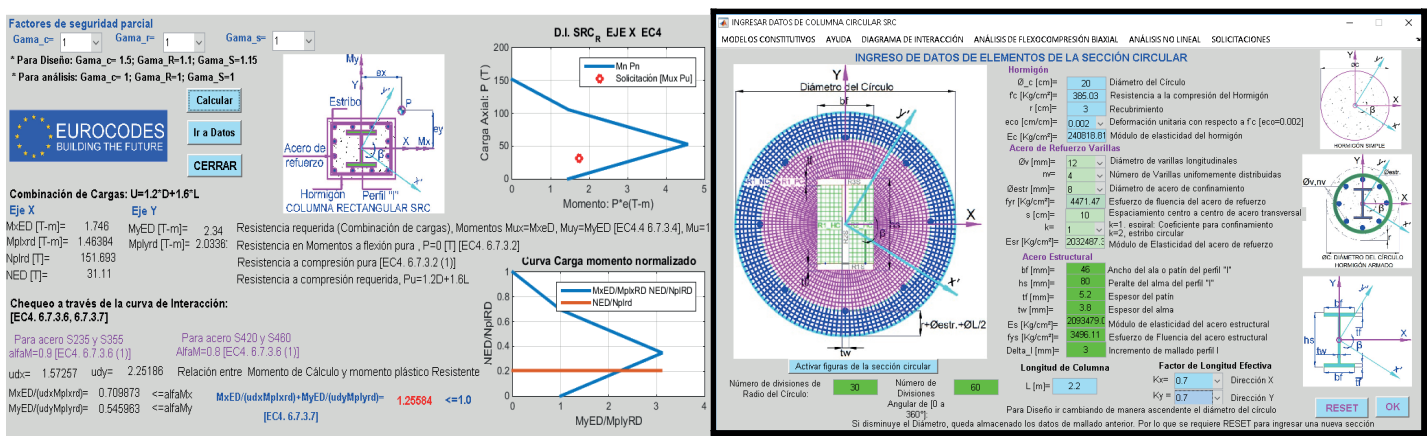

Figura 2: Compatibilidad de esfuerzos y deformaciones en una sección plana. Columna SRC Rectangular y Circular, método de la fibra

compresión. Se considera que las secciones permanecen planas, durante la aplicación de la carga.

\subsection{Diagramas de Interacción Según Criterios de los Códigos $\mathrm{ACl} 318$, AISC-LRFD y Método de Fibras.}

El diagrama de interacción a flexocompresión de una columna SRC permite conocer la capacidad en momentos flectores y carga axial, los mismos que son construidos en base a criterios con los cuales trabajan los códigos ACl 318, AISC LRFD 360 y Método de las Fibras. Se realizan estos diagramas tanto para las columnas circulares como rectangulares. A manera de ejemplo se ilustra en la figura 3 un diagrama que corresponde a las columnas rectangulares.

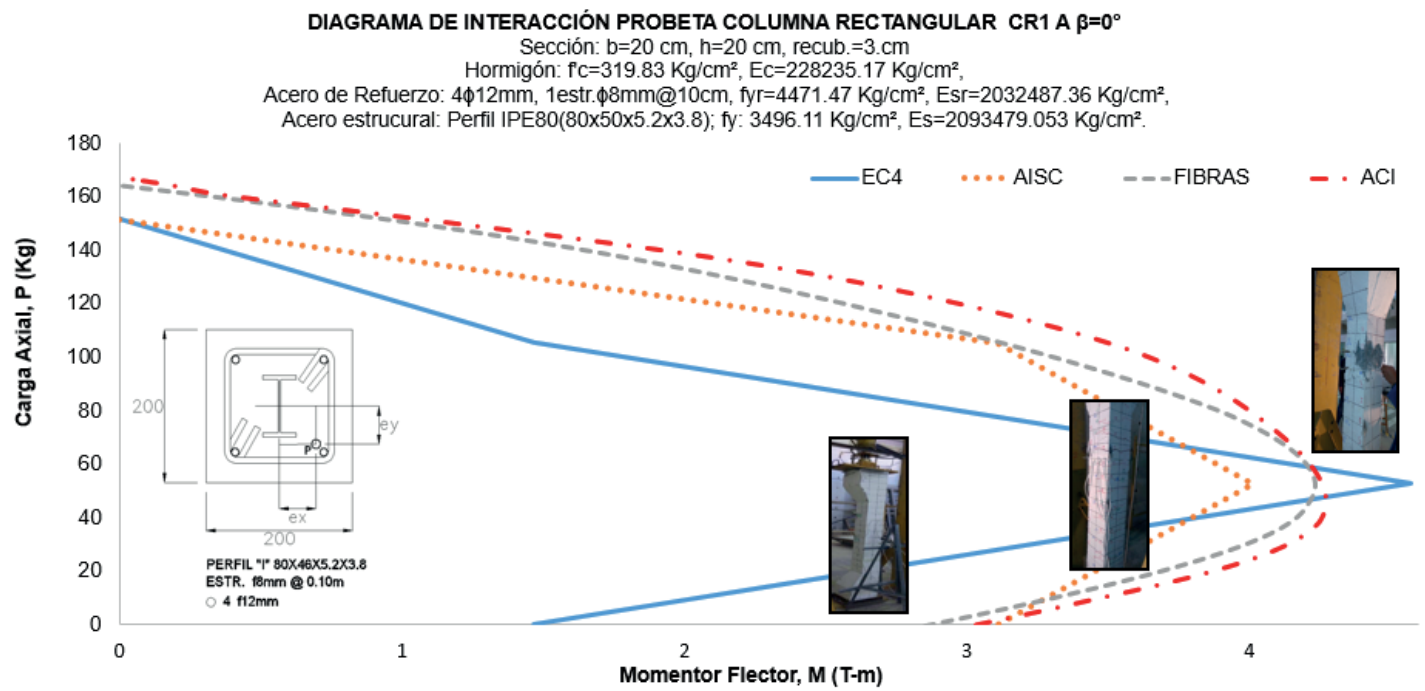

Figura 3: Diagramas de Interacción para columnas rectangulares CR1 y CR2 


\subsection{Curvas Carga Desplazamiento Experimentales y Teóricas}
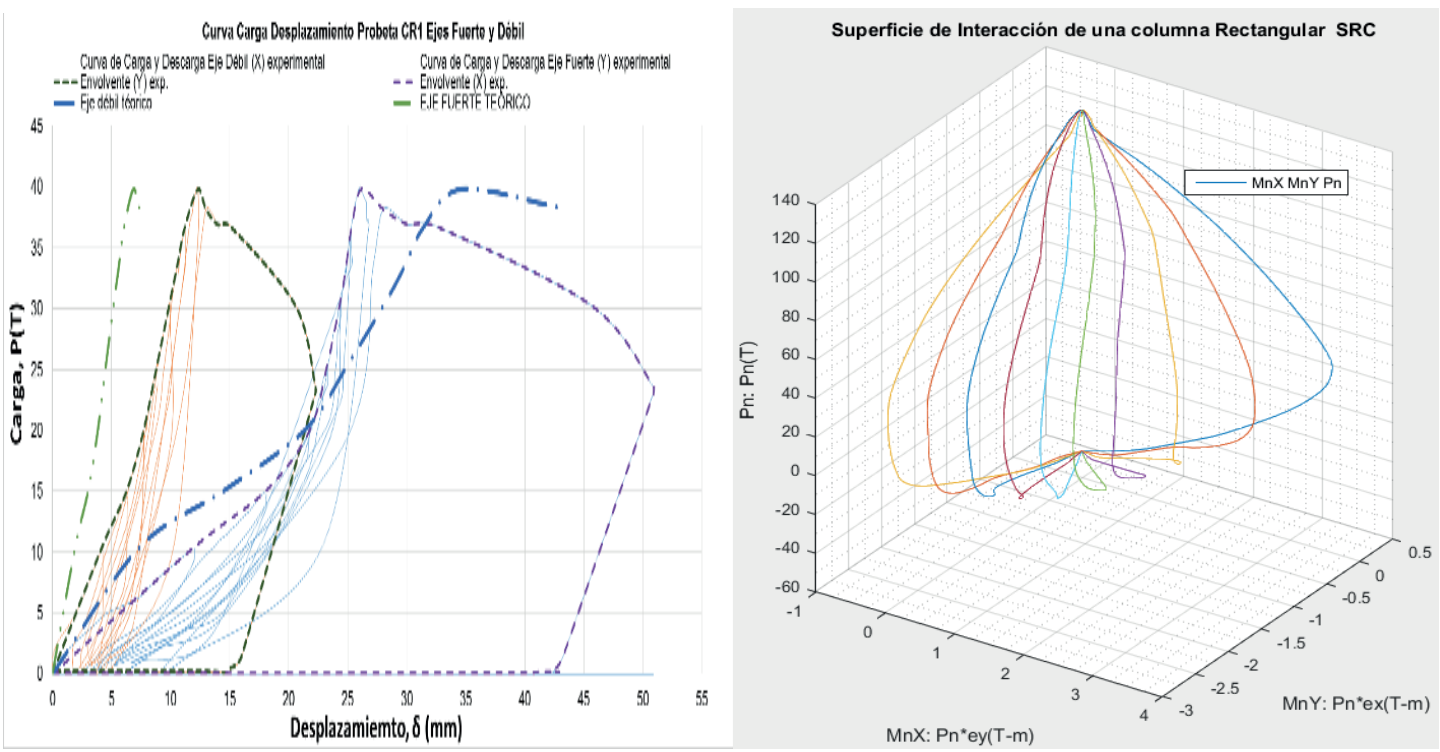

Figura 4: Curvas Carga Desplazamiento para una probeta rectangular ensayada

Se realizan ensayos destructivos aplicando una carga axial de compresión a una determinada excentricidad. Esta carga axial se aplica a cada columna con la modalidad de carga cíclica, esto se realiza con la finalidad de obtener valores de rigideces laterales $\mathrm{Kx}$ y Ky, y verificar el comportamiento numérico de las mismas en cada ciclo de carga.

Además, mediante la curva carga desplazamiento experimental, como se muestra en la figura 4, se puede determinar el valor de la rigidez flexión (EI) obtenida para cada ciclo de carga y que está en función de la carga axial $P$, la longitud $L$ de la columna, la excentricidad y el desplazamiento $\delta$ medido en el centro de la columna. Se construyen estas curvas para cada una de las columnas estudiadas. A manera de ejemplo expondremos la curva Carga-desplazamiento de una de las probetas cilíndricas.

Los valores de rigidez a flexión de las columnas (EI) en cada ciclo de carga son determinados en base a la ecuación de la viga columna deducida. Como ejemplo se muestra la figura 5 para una de las columnas rectangulares ensayadas. Estos valores estas en función de la carga axial aplicada durante el ensayo. Para un ensayo de carga y momentos biaxiales la resistencia a compresión axial de agotamiento es menor en un 50\% que la carga nominal Pn que se calcula con el método del AISC LRFD 360. 


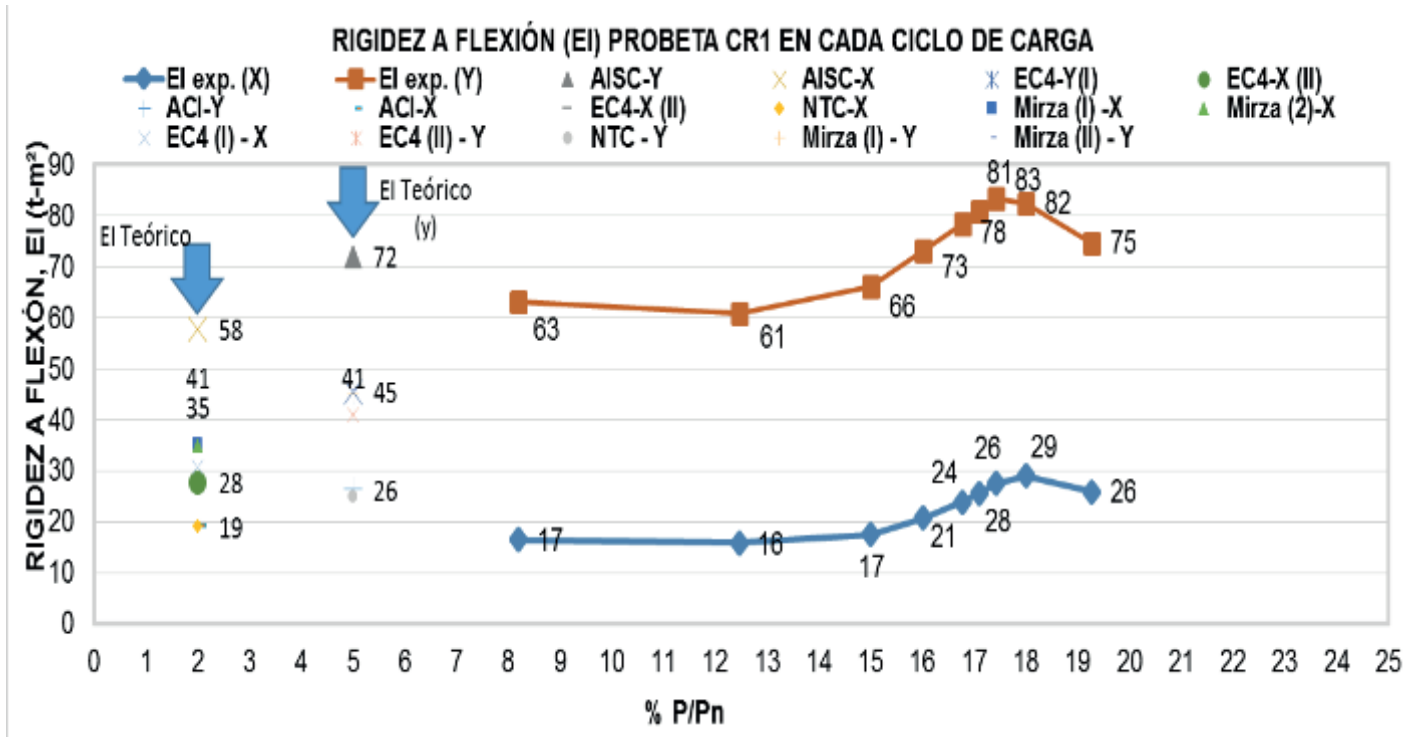

Figura 5: Rigidez a flexión de una de las columnas rectangulares ensayadas

\subsection{Factor de Ductilidad a Desplazamiento de las Muestras}

Se procede a determinar la ductilidad de las probetas $(\mu)$. Considerando que la carga de plastificación $\mathrm{Pp}$, se determina en donde se presenta un cambio de pendiente brusco en la curva carga versus desplazamiento.

TABLA 4: Ductilidad a desplazamiento en el eje débil

\begin{tabular}{|c|c|c|c|c|c|c|c|}
\hline Probeta & $\delta y(\mathrm{~mm})$ & Py (kN) & $\delta \mathbf{p}(\mathrm{mm})$ & $\mathrm{Pp}(\mathrm{kN})$ & $\delta \mathbf{u}(\mathrm{mm})$ & Pu (kN) & $\mu \mathbf{X}$ \\
\hline CR1 & 18.09 & 150.6 & 23.48 & 523.4 & 49.51 & 523.4 & 2.11 \\
\hline $\mathrm{CR}_{2}$ & 3.47 & 150.7 & 7.67 & 400.5 & 20.26 & 400.5 & 2.6 \\
\hline $\mathrm{CC}_{1}$ & 5.86 & 300.0 & 5.28 & 256.5 & 11.34 & 256.5 & 2.1 \\
\hline $\mathrm{CC}_{2}$ & 6.98 & 305.5 & 10.23 & 519.3 & 21.45 & 519.3 & 2.10 \\
\hline
\end{tabular}

La ductilidad desplazamiento de cada miembro se obtuvo trazando una línea paralela al eje de las abscisas de la curva carga desplazamiento de cada probeta, en el punto donde hay un cambio brusco de pendiente, punto que define la carga axial plástica experimental de las secciones. Los desplazamientos plástico y último son encontrados en base de los cuales se calcula el coeficiente de ductilidad a desplazamiento. Se obtienen ductilidades mayores a dos, es decir miembros de estructuras con una ductilidad adecuada, valores que han sido obtenidos en investigaciones afines. 
TABLA 5: Ductilidad a desplazamiento en el eje fuerte

\begin{tabular}{|c|c|c|c|c|c|c|}
\hline Probeta & $\delta y(\mathrm{~mm})$ & Py (kN) & $\delta \mathbf{p}(\mathrm{mm})$ & $\mathrm{Pp}(\mathrm{kN})$ & $\delta \mathbf{u}(\mathrm{mm})$ & Pu (kN) \\
\hline CR1 & 18.09 & 150.6 & 8.84 & 523.4 & 22.20 & 523.4 \\
\hline CR2 & 3.47 & 150.7 & 4.42 & 400.5 & 10.96 & 400.5 \\
\hline $\mathrm{CC}_{1}$ & 5.86 & 300.0 & 3.18 & 256.5 & 11.33 & 256.5 \\
\hline$C_{2}$ & 6.98 & 305.5 & 8.14 & 519.3 & 15.78 & 519.3 \\
\hline
\end{tabular}

TABLA 6: Ductilidad a desplazamiento en el eje vertical

\begin{tabular}{|c|c|c|c|c|c|c|c|}
\hline Probeta & $\delta y(\mathrm{~mm})$ & Py (kN) & $\delta \mathrm{p}(\mathrm{mm})$ & $\mathrm{Pp}(\mathrm{kN})$ & $\delta \mathbf{u}(\mathrm{mm})$ & $\mathrm{Pu}(\mathrm{kN})$ & $\mu \mathbf{Z}$ \\
\hline CR1 & 3.73 & 150.6 & 4.29 & 523.4 & 11.81 & 523.4 & 2.75 \\
\hline CR2 & 1.43 & 150.7 & 4.26 & 400.5 & 10.39 & 400.5 & 2.44 \\
\hline $\mathrm{CC}_{1}$ & 1.02 & 300.0 & 0.98 & 256.5 & 4.80 & 256.5 & 4.90 \\
\hline$C 2$ & 1.45 & 305.5 & 1.8 & 519.3 & 7.39 & 519.3 & 4.11 \\
\hline
\end{tabular}

\section{Conclusiones}

1. La programación del cálculo del diagrama de interacción por el método de la fibra formulado en el presente trabajo permite calcular la capacidad de las columnas manifestada en esfuerzos, fuerzas axiales y momentos flectores a un determinado eje de inclinación del eje neutro.

2. Se utiliza el programa de las fibras para calcular el diagrama de interacción de las probetas a ensayar, el mismo que permite la predicción de la carga por agotamiento por flexocompresión biaxial.

3. Respecto a la predicción de carga axial de diseño de las probetas se toma un valor asumido de coeficiente de esbeltez de $\mathrm{K}=0.70$, considerando que la columna está empotrada en la cabeza y apoyada en el pie. Al revisar la carga de agotamiento en el diagrama de interacción corresponde al caso de una columna intermedia y se puede encontrar que la falla se produce por el deterioro del hormigón.

4. Al graficar las curvas carga desplazamiento se obtiene un mayor desplazamiento en la dirección más débil de la probeta. La comparación de valores de desplazamientos en el eje débil respecto al eje fuerte corresponde para la columna rectangular CR1 al $230 \%$, para la columna rectangular CR2 es $109 \%$, en la columna circular CC1 es $80 \%$ y para la columna circular CC2 es $222 \%$. Las cuatro columnas ensayadas fallaron por desmoronamiento del hormigón en la zona de compresión. 
5. Al comparar los valores de la resistencia nominal de momentos flectores, obtenida del diagrama de interacción por el método de la fibra, el porcentaje de acierto en la predicción de la carga para la probeta rectangular CR1 es 137\%, para el eje débil y $214 \%$ para el eje fuerte, para la probeta rectangular CR2 es $138 \%$ en momentos para el eje débil y $216 \%$ para el eje fuerte. Para la probeta circular $\mathrm{CC}_{1}$ se tiene el $98 \%$ de acierto para el eje débil y $169 \%$ para el eje fuerte, y para la probeta circular CC2 se obtuvo el 98\% de acierto en momentos para el eje débil y 166\% para el eje fuerte. Por lo tanto, los resultados del diagrama de interacción normativos para la predicción carga axial y momentos flectores de miembros SRC ofrecen valores de alta confianza. Ciertamente, los modelos como los del $\mathrm{ACl} 318$ y del método de la fibra, los cuales trabajan con modelos bilineales y con modelo constitutivos de los materiales, consideran una seguridad apropiada de los diseños de miembros SRC

6. Los resultados de factor de ductilidad a desplazamiento proveen valores que son mayores a dos, por lo que puede se concluye que las columnas ensayadas presentan comportamiento de miembros dúctiles, y corresponden a valores obtenidos por investigaciones similares.

\section{Referencias}

[1] American Concrete Institute (2014) 'Building Code Requirements for Structural Concrete (ACl 318-14)". An ACI Standard. Farmington Hills, MI 48331 USA. 509pp.

[2] American Institute of Steel Construction. (2016) "Specification for Structural Steel Buildings" ANSI/AISC 360-16. An American National Standard. Chicago, Illinois 60601-1802. 612pp.

[3] European Committee for Standardization (2009). "Design of composite steel and concrete structures Part 1-1: General rules and rules for buildings". EUROCODE 4. Brussels. Belgium. 118pp.

[4] McCormac, J.C.; Csernak, S. (2013) "Diseño de estructuras de acero". $5 a$ Edición. Editorial Alfaomega. 736pp. ISBN: 9786077075592.

[5] Segui, W.T. (2013) "Steel design". Fifth Edition. University of Memphis. Editorial Global Engineering. 752pp. ISBN-13: 978-1-111-57600-4. 


\section{Authorization and Disclaimer}

Authors authorize ESTEC to publish the paper in the conference proceedings. Neither ESTEC nor the editors are responsible either for the content or for the implications of what is expressed in the paper. 